\title{
A NEW WAY FOR DOD TO DO BUSINESS: THE J-MASS MARKETPLACE
}

\author{
William K. McQuay \\ Wright Laboratory
}

\begin{abstract}
It is current DoD policy to use commercial off-the-shelf software whenever it meets DoD requirements. The application of this policy to modeling and simulation has resulted in the concept of "The Joint Modeling and Simulation System (I-MASS) Marketplace."
\end{abstract}

J-MASS is designed as an Open Systems Architecture with the capability for the Simulation Support Environment (SSE) to be expanded by the addition of site specific software. In the "J-MASS Marketplace" industry will build commercial tools to work with J-MASS and individual organizations will license what they need for their particular site. The J-MASS SSE is a framework or backplane into which everything else piugs. A J-MASS product release would have the core capabilities, but the unique needs of various organizations would be satisfied by industry.

This paper addresses how the $\mathrm{J}$ MASS Marketplace could work and how compliance can be defined. It will outline opportunities for industry in both building software for the Marketplace and in defining the Marketplace concept.

\section{Introduction}

In these times of increased fiscal constraint often resulting in corporate downsizing, DoD, like other organizations, is undergoing many changes in structure, management, and process. Two changes are germane to this paper: use of commercial standards and increased use of modeling and simulation.

One change is to adopt corporate business practices where possible. Commercial and industry standards will be used in lieu of military standards. For example, Secretary of Defense Perry's June 29, 1994 memorandum, "Specification \& Standards -- A New Way of Doing Business," states that military standards will only be used "as a last resort, with an appropriate waiver" when specifying military acquisition requirements. Secretary Perry's memorandum encourages practices that satisfy the Department's need to build high quality systems that meet requirements at affordable costs and in a timely manner. This includes practices which support the development of Defense Software. The new DoD policy is to use commercial off-the-shelf (COTS) software whenever it meets DoD requirements. If COTS software is not available to satisfy requirements, then DoD must develop unique software to meet its needs.

A second change is increased use of modeling and simulation (M\&S) throughout all functional areas in DoD. Use of $M \& S$ is already widespread. Various studies have estimated that DoD spends up to $\$ 1$ billion annually on digital modeling (not including training and realtime simulators) . Because of increased technical capability and increased fiscal constraint, the M\&S market will continue to expand as other budgets are reduced. In the FY95 DoD Authorization Act, Congress explicitly emphasized and promoted the use of modeling and simulation in training, development of doctrine and acquisition. Dr. Anita Jones, Director of Defense Research and Engineering, has rated information technology and M\&S as two of the top three Defense priorities. Edwin Dorn, Undersecretary of Defense for Personnel and Readiness, in prepared remarks entitled "Reinventing DoD: More Performance, Less Cost", cited distributed simulation technology in DoD 
as among the best examples of reinventing government - offering better service and better performance, for less taxpayer money.

The importance of $\mathrm{M} \& \mathrm{~S}$ has resulted in DoD Directive 5000.59, "DoD Modeling and Simulation Management", which provides for a DoD M\&S Master Plan. As outlined in the draft plan, DoD intends to establish a common high-level simulation architecture to ensure the appropriate interoperability of live, virtual and constructive simulations, and their interface with command, control, communications, computers and intelligence systems. All ongoing DoD M\&S projects/programs will be reviewed for the feasibility of adopting the highlevel architecture. The goals of the architecture include: interoperability, reuse, portability, distributed operation, legacy operation, scalability, broad applicability, technological evolvability, and maximum feasible use of COTS and government off-the-shelf (GOTS) products.

$J$-MASS is envisioned as the Air Force common modeling and simulation architecture to be used as the "engine of the EW Test Process". As the DoD High Level Architecture evolves, J-MASS will comply with those standards. In light of these events, Wright Laboratory and the J-MASS Program Office (ASC/XREM) are developing the concept of the $J$ MASS Marketplace, where industry would build commercial tools to work with J-MASS in response to real customer demand. From its inception, JMASS was designed as an Open Systems Architecture with the capability for the Simulation Support Environment to be expanded by the addition of site specific software, including COTS.

\section{J-MASS -- A GOTS Framework for COTS}

Under the Marketplace concept, the J-MASS SSE serves as a GOTS framework for the addition of third party applications. A J-MASS product release will provide core capabilities which will meet the needs of many J-MASS users. But the unique needs of many organizations would be satisfied by industry developed J-MASS applications meeting specific requirements. The Marketplace concept avoids the high cost of DoD-wide licenses and permits commercial market competition to satisfy individual organizational requirements. Broad DoD and Service requirements could be satisfied by core J-MASS capabilities or, in many cases, by choices from multiple commercial tools that would be J-MASS compliant. DoD has insufficient resources to purchase DoDwide licenses for the multiplicity of unique and individual products required for all DoD and Service organizations. Instead J-MASS becomes a new market for application developers where the field organizations buy the exact product they need. Companies will have a new arena for sales of commercial products (tools and eventually even model parts) compatible with J-MASS. Commercial applications could carry a "J-MASS Compliant" label much like the commercial logos found on PC products today. Many existing tools on the commercial market and widely used by industry could easily be made compatible with the SSE. The commercial tools can add functionality across all modes of the SSE: visual programming toolkits for the Develop, icon-based visually assembly for Assemble, geographical information system and map tools for Configure, runtime monitoring visualization for Execute, and word processing and presentation graphics for Post Process. Such tools are more affordably and efficiently created by the commercial sector and can be maintained at low cost by a broad customer base. All J-MASS sites do not need all the available tools. It is not necessary for DoD to license everything for everybody. The everyday user is the best judge of what the user organization requires. The user organization can chose which commercial products to license from the many alternatives potentially available in the J-MASS 
Marketplace. With formal definition of a tool application programming interface (API) and other standards, products could be plugged into the SSE seamlessly.

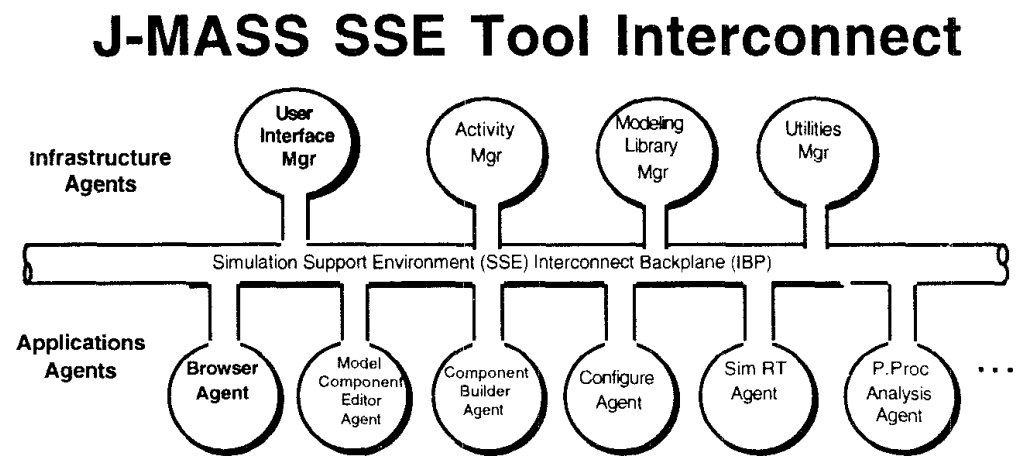

Figure 1. Notional Depiction of J-MASS SSE Tool Interconnect

\section{Overview of the J-MASS SSE Architecture}

A notional depiction of the SSE architecture is shown in Figure 1. A Graphical User Interface (GUI) provides a MOTIF style push-button user interface which integrates the toolset. A Common Object Request Broker Architecture (CORBA)-like brokered software service backplane called the Interconnect Backplane (IBP) provides for control of tools, data flow, and input/output files. Each tool (or agent), registers with the backplane and no tool requires a priori knowledge of any other tool (or service) in the system. In J-MASS Release 2.0, the SSE IBP itself resolves the location of the service provider. In J-MASS Release 3.0, the Activity Manager on the SSE IBP will provide this function. Files or information sets are maintained within a POSIX-compliant Name Manager component of a Modeling Library.

The J-MASS IBP has a multilayered design as shown in Figure 2. The design includes layers for the network, Berkeley Sockets, Unified Message Passing, and the IBP API. The Unified Message Passing layer provides industry standard communication protocols such as Unit Datagram Protocol (UDP) for real-time and Transmission Control Protocol (TCP) for assured message passing and hides any machine dependencies.

A Tool API interfaces to the IBP and provides tool configuration files, messaging to the Activity Manager, and tracks all tools in use for the session. 


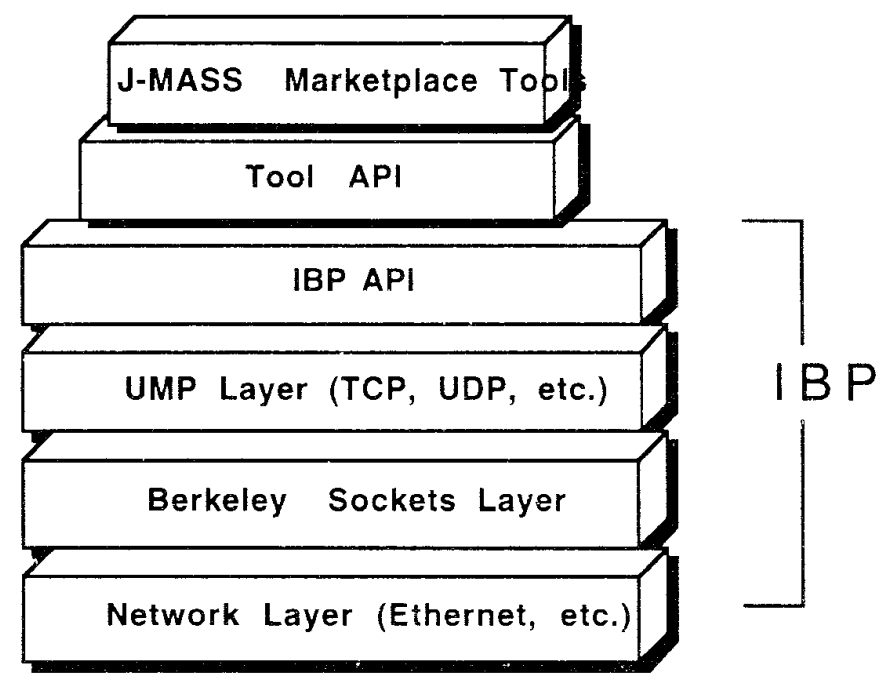

Figure 2. IBP Layers

The J-MASS architecture is based on many commercial standards, such as CORBA, POSIX, MOTIF, and TCP, which will make a marketplace even more viable.

\section{What is J-MASS Compliance for Tools?}

Compliance is an important issue since a fundamental tenet of J-MASS is that most capabilities, be it models or tools, will not be provided by a central J-MASS organization, but by the J-MASS user community or the commercial sector. J-MASS capabilities are of two fundamental types: tools and simulation models. First, let us review some definitions. DODD 5000.59 defines a model as a physical, mathematical, or otherwise logical representation of a system, entity, phenomenon, or process. A simulation is a method for implementing a model over time. In the context of J-MASS, a simulation model is a capability that connects into the J-MASS simulation engine, i.e., the Simulation Runtime Agent (SRA), and interacts with other models. A tool is a software package that builds a J-MASS compliant model, acts on a J-MASS compliant model, or acts on data either from or for a J-MASS compliant model or tool.

There are two aspects to J-MASS compliance: support and structurai. Support is the data necessary for the software to be used by a member of the J-MASS community. Structural compliance has to do with how the software initiates execution and how it communicates with other J-MASS compliant software. Structurally JMASS compliant toois must

- Receive User Commands via the J-MASS Graphical User Interface

- Initiate execution across the J-MASS Tool Backplane

- Communicate through the J-MASS Tool Backplane

- Handle data via the J-MASS Modeling Library capability

To insure supportability, J-MASS compliant tools must: 
- Execute on the J-MASS target workstations

- Have a Software User's Manual

- Have a Test Report with test drivers

- Provide an abstract and facet terms for the M\&S Reuse Library clearinghouse

- Provide files for hypermedia on-line J-MASS Help in HTML format

- Have an available support structure so that J-MASS users can get assistance when required

- Have a model (or some other piece of software) that can be used by the JMASS Customer Support Center to isolate anomalies between the new tool and J-MASS

The goal for J-MASS is field extensibility - the ability for the user to add various COTS and GOTS tools as required for that user site and to be able to do it as easily as a personal computer or Macintosh user adds word processor software to a home or office computer. Field extensibility would require several enhancernents to the J-MASS SSE. These enhancements would include capabilities to dynamically maintain the JMASS Graphical User Interface, incorporate new COTS applications or delete existing ones, setup application environments, a customize feature to tailor each mode and move application icons between windows, and to dynamically create new Modeling Library data types for new applications. A Tool Developer's Manual would be required to provide all necessary information for a third party to design, develop, test and integrate a tool into the J-MASS architecture. The manual would include requirements and methodology for interface and integration, message and control structures, documentation, and testing.

\section{Industry Participation}

The J-MASS Marketplace is a new concept and potentially large market area for the future. Industry is invited to assist in developing how the J-MASS Marketplace will operate, what standards need be defined, and how compliance testing should be implemented. Industry has the opportunity to participate in the definition of this new market concept. Send comments, suggestions, ideas for the types of commercial tools, or your desire to participate in future J-MASS Symposia discussions of the J-MASS Marketplace to the J-MASS Program Office, ASC/XREM, Wright-Patterson AFB, OH 45433. 